\title{
Ist Beihilfe zum Suizid mit dem ärztlichen Ethos vereinbar?
}

\author{
In den letzten Jahrzehnten fand ein gesellschaftlicher Wertewandel in Bezug \\ auf das Lebensende statt. In der öffentlichen Diskussion verlor die Lebensver- \\ längerung ihre früher absolute Priorität zugunsten von Lebensqualität und \\ dem Anspruch auf einen selbstbestimmten Tod. Nun wird immer mehr einge- \\ fordert, diesen Anspruch auch praktisch umzusetzen. Diese Entwicklung soll \\ als Herausforderung für das ärztliche Ethos kritisch dargestellt und diskutiert \\ werden.
}

\section{Stefan Freidel}

Ich widme den Artikel in Dankbarkeit Herrn Prof. Dr. F. J. Illhardt, Zentrum für Ethik und Recht in der Medizin, Albert-Ludwigs-Universität Freiburg i. Br. Ausserdem danke ich meiner Frau, Ursula Becker-Freidel, für das Korrekturlesen und die kritische Diskussion des Artikels.

1 Student JC. Hospizbewegung und Dilemmata der Sterbehilfe. In: Illhardt FJ, Heiss HW, Dornberg M (Hrsg.). Sterbehilfe - Handeln oder Unterlassen? Stuttgart, New York: Schattauer; 1998. S. 91-7.

2 Titz C. Der Kampf gegen den Tod. OnlineFocus, 16.12.07.

3 Ariès P. Geschichte des Todes. 2. Auflage. München: dtv; 1987.

4 Baumann-Hölzle R. Selbsttötung als Menschenrecht - ethische Überlegungen zu einem gesellschaftlichen Klimawandel. Schweiz Ärztezeitung. 2007; 88(35):1446-51.

Korrespondenz:

Dr. med. Stefan Freidel

Freiestrasse 4

CH-8610 Uster

praxis@freidel.ch
Sterblichkeit ist ein zentrales Thema menschlicher Existenz, das von jeher starke Ängste ausgelöst hat [1]. Die Angst vor dem eigenen Tod ist eine Triebfeder des ärztlichen Handelns, das gelegentlich «Kampf mit dem Tod» genannt wird [2]. Die therapeutischen Möglichkeiten sind jedoch so sehr angewachsen, dass ihr Einsatz nicht nur Leid vermindern, sondern selbst Leid verursachen kann. Bekämpfte die Medizin früher vor allem akute Erkrankungen, traten zunehmend chronische Leiden in den Vordergrund, die mit immer invasiveren und aufwendigeren Mitteln bekämpft wurden. Sie verstärken die Auswirkungen der bereits seit langem beobachtbaren Verlagerung des Sterbens aus dem häuslichen Umfeld ins Krankenhaus [3]. Das meist rasche Sterben zuhause im Kreise von Familie, Freunden und Bekannten wich einer Vereinsamung im Krankenhaus. Einsamkeit stellt nicht umsonst eine zentrale Angst Sterbender dar [1]. Hinzu kommt die Angst vor Schmerzen durch Krankheit, therapeutische Eingriffe und unzureichende Analgesie.

Ausgelöst durch die Schicksale von über Monate im Wachkoma liegenden Menschen und den in die Länge gezogenen Sterbeprozessen von Staatsmännern wie Tito und Franco wurde das ärztliche Handeln am Lebensende von der Öffentlichkeit zunehmend kritischer betrachtet. Der mit der wachsenden Medikalisierung des Lebensendes einhergehende Autonomieverlust sensibilisierte für das ärztliche Vorgehen, dem nicht mehr ohne weiteres die optimale Wahrnehmung der Patienteninteressen zugetraut wird. Die Angst davor, das Leben könne entgegen dem eigenen Willen verlängert werden und dadurch unzumutbares Leid, vor allem Schmerzen und Einsamkeit verursacht werden, führte zu der Forderung nach einem autonomen und menschenwürdigen Sterben.

\section{L'assistance au suicide est-elle compatible avec l'éthique médicale?}

Au cours des dernières décennies, la société a subi un changement de valeurs en ce qui concerne la fin de vie. Les débats publics sur la question de la prolongation de la durée de vie, prioritaire dans le passé, a perdu de l'importance au profit de la qualité de vie et de l'exigence de libre détermination de sa propre mort. Désormais, de plus en plus de voix se font entendre pour appliquer concrètement cette exigence. Cette évolution, qui est un défi en matière d'éthique médicale, doit être présentée et discutée de manière critique.

Andererseits weckt die Forderung nach einer Beendigung des Lebens selbst Ängste vor einer unfreiwilligen Verkürzung des Lebens. Damit einhergehend wird ein Dammbrucheffekt befürchtet [4]. Was jetzt als Ausnahme in besonders schwerwiegenden Fällen eingefordert wird, könnte in der Zukunft in immer leichteren Fällen Usus werden. Ärzte, die dann keine Sterbebegleitung durchführen, könnten zunehmend unter Druck geraten, diese anzubieten. Eine weitere Gefahr ist, dass nicht der Patientenwillen, sondern die ärztliche Abwägung der mutmasslichen Lebensqualität des Patienten Entscheidungsgrundlage werden könnte. Gesundheitsökonomische Anreizsysteme, insbesondere Kosteneinsparungen durch Sterbehilfe, könnten in die Entscheidungsfindung einfliessen. Ob es jedoch wirklich 
zu einem Dammbrucheffekt kommt, der das ärztliche Ethos aufweicht, ist fraglich [5]. Auch bedeutet es nicht, dass allfällige Fehlentwicklungen unumkehrbar sind. Diese Gefahren sprechen denn auch nicht in erster Linie für ein generelles Verbot von Sterbehilfe, sondern für eine Begrenzung auf Extremfälle [6].

\section{Die Herausforderung für die Ärzteschaft}

Dem zunehmenden gesellschaftlichen Bedürfnis nach assistiertem Suizid stehen auf ärztlicher Seite Bedenken gegenüber, die Rolle als Sterbehelfer sei nicht mit dem ärztlichen Ethos vereinbar [7]. Es sei die Aufgabe des Arztes, Leben zu erhalten, und nicht, es zu beenden. Hier stehen sich die Sichtweise des Arztes und des Patienten konflikthaft gegenüber. Selbst wenn es aus ethischer Sicht keinen relevanten Unterschied zwischen aktiven und passiven Massnahmen gibt [8], ergibt sich dabei für die ausführenden Ärzte ein psychologischer Unterschied. Die subjektive Verantwortung ist bei aktiven Handlungen, die zum vorgezogenen Tode des Patienten führen, grösser. Die Ärzteschaft positioniert sich nicht zuletzt deshalb eher skeptisch bis ablehnend zu der dargestellten gesellschaftlichen Entwicklung. Wenn es ihr aber nicht gelingt, diese beiden Standpunkte in ein fruchtbares Spannungsverhältnis zu setzen und dieses weiterzuentwickeln, besteht die Gefahr, dass Dritte Entscheidungen fällen, die nicht im Sinne der Ärzteschaft sind. Ein Beispiel dafür ist Belgien, wo die Ärzteschaft von der gesellschaftlichen und juristischen Entwicklung bezüglich der Sterbehilfe überrollt wurde [9]. Auch um den Schutz des Lebens zu verbessern, sollte ein Auseinanderdriften von Standesethik und ärztlicher Praxis und damit eine Doppelmoral mit intransparenten Praktiken verhindert werden. Bei ihnen besteht ein Risiko für Fehlentscheidungen durch mangelnde Rückbindung an ethische und fachliche Diskussionen, das aus Sicht des Autors grösser ist als bei differenzierteren ärztlichen Handlungsoptionen.

In der Schweiz haben sich Sterbehilfevereine der Thematik angenommen. Die Ärzte könnten sich freuen, dass sich andere um dieses strittige Gebiet kümmern und ihnen die Rolle erhalten bliebe, nicht schaden $\mathrm{zu}$ wollen (primum non nocere). Verschiedene Entwicklungen der jüngsten Zeit im Bereich des assistierten Suizids bereiten jedoch Anlass zur Sorge und lassen einen breiten gesellschaftlichen Diskurs notwendig erscheinen. Aus Sicht des Autors haben die Ärzte eine Verpflichtung gegenüber der Gesellschaft, dazu beizutragen, dass in ihr verantwortlich mit dem Lebensende umgegangen wird.

\section{Was können Ärzte zu einem verantwortungsvollen Umgang mit dem Tod beitragen?}

Hierbei stehen zwei Optionen zur Verfügung. Mit Exit und Dignitas geht die aktuelle Entwicklung dahin, Sterbebegleitungen ausserhalb des medizinischen Systems anzusiedeln. Gerade das Vorgehen von Dignitas, das immer wieder ausführlich in den Medien dokumentiert wird, macht die Wichtigkeit von gesellschaftlich akzeptierten und breit abgestützten Vorgehensweisen und Regeln deutlich, um das Risiko von Fehlentwicklungen $\mathrm{zu}$ reduzieren. Gerade wenn es um Fragen von Leben und Tod geht, braucht es ein qualitativ hochstehendes, kontrolliertes und transparentes Angebot. Bei der Entwicklung und Umsetzung solcher Regeln und Standards ist die medizinische Expertise wichtig: Das Stellen von Diagnose und Prognose, das Aufzeigen von Behandlungsoptionen und das Diagnostizieren von Begleitfaktoren wie Depressionen sind genuin ärztliche Aufgaben. Allerdings sollten in den vorbereitenden und ausführenden Gremien neben Ärzten zusätzlich andere Berufsgruppen, insbesondere Juristen und Ethiker, vertreten sein [9]. Auch der Beizug weiterer relevanter Berufe, z. B. Theologie, Pflege und Sozialarbeit, ist zu diskutieren. Diese Variante geht davon aus, Ärzte zumindest nicht per se zu Experten für Sterbewillige zu machen und insbesondere einen entsprechenden Ausschliesslichkeitsanspruch der Medizin zu vermeiden. Ihre Stärke liegt darin, die Verantwortung für das sich wandelnde gesellschaftliche Bedürfnis an die Gesellschaft zurückzudelegieren, ohne sich dem dafür notwendigen Diskurs zu entziehen.

Im Folgenden soll untersucht werden, ob sich die Beihilfe zum Suizid wirklich so schlecht mit der ärztlichen Rolle verträgt, wie bisweilen argumentiert wird. Trotz der zunehmenden Hospitalisierung des Todes soll nicht übersehen werden, dass immer wieder Hausärzte ihre Patienten während langer Krankheitsphasen bis zum Tode begleiteten. Unter der Hand wird auch vereinzelt von durchgeführten Sterbehilfemassnahmen berichtet. In den teilweise sehr vertrauensvollen Langzeitbehandlungen älterer Menschen sollte es möglich sein, die Einstellung gegenüber dem bevorstehenden Tod über die Zeit hinweg zu erfassen und mit dem Patienten in einen Dialog darüber einzutreten. Dabei können die Ansichten zur Prognose und Handlungsoptionen diskutiert und immer wieder abgewogen werden. Dadurch kann das Gefühl des Ausgeliefertseins an ein gnadenloses Schicksal relativiert werden. Meist wird dies zu einer Erleichterung und zu einer Distanzierung von Todeswünschen führen. Erfahrungs- neue Rolle für Europas Ärzteschaft? Schweiz Ärztezeitung. 2008; 89(10):411-5. 
gemäss steht hinter Todeswünschen oft die versteckte Bitte nach der Linderung eines Leidens, z. B. von Schmerzen, Isolation oder Ängsten. Ergibt sich jedoch eine der sehr seltenen Situationen, in denen der selbstbestimmte, vorgezogene Tod auch dem wohlwollend-kritischen Arzt als die beste Möglichkeit erscheint, gebietet es der Respekt gegenüber dem Patienten, diesen Willen anzuerkennen [10]. Wer ihm nicht selbst nachkommen will, sollte zumindest seinen Patienten eine würdevolle und qualitativ gute Alternative durch Dritte anbieten.

\section{Die Betonung des ganzen Menschen stellt ein gewichtiges} Argument für die Sterbebegleitung als Bestandteil des ärztlichen Repertoires dar.

10 Freidel S. Ärztliches Handeln am Lebensende. APIS. 1997;X:76-83.

11 Dornberg M. Bemerkung zur Struktur philosophischer Argumentationen im Kontext der Sterbehilfe-Diskussion. In: Illhardt FJ, Heiss HW, Dornberg M (Hrsg.). Sterbehilfe - Handeln oder Unterlassen? Stuttgart, New York: Schattauer; 1998. S. 65-70.

12 Dekkers W. Eine unversehrte Heimkehr. Sterbehilfe und Hospizbewegung in den Niederlanden. In: Illhardt FJ, Heiss HW, Dornberg M (Hrsg.). Sterbehilfe Handeln oder Unterlassen? Stuttgart, New York: Schattauer; 1998. S. 99-111.

13 Imhof AE. Die Kunst des Sterbens Wie unsere Vorfahren sterben lernten. Stuttgart: Hirzel; 1998.

14 Raggenbass R, Kuhn HP. Kein Menschenrecht auf ärztliche Suizidbeihilfe. Schweiz Ärztezeitung. 2007;88(1):455-6

15 Illhardt FJ. Das Leben des Sterbenden - eine Herausforderung an Selbstverwirklichung und Selbstbestimmung. In: Illhardt FJ Heiss HW, Dornberg M (Hrsg.) Sterbehilfe - Handeln oder Unterlassen? Stuttgart, New York: Schattauer; 1998. S. 113-9.
Die Betonung des ganzen Menschen unter Einschluss seiner persönlichen und sozialen Bedürfnisse stellt ein gewichtiges Argument für die Sterbebegleitung als Bestandteil des ärztlichen Repertoires dar [11]. Wie Dornberg betont, geht der Begriff «Beziehung» über die von philosophischer Seite betonte Erkenntnis des Patientenwillens und die gegenseitige Achtung hinaus. Er beinhaltet die «Möglichkeit gegenseitiger Veränderung und Ergänzung, Verantwortung» und eine gewisse Sympathie. Vom Arzt hängt es ab, ob der Patient seine Todeswünsche konkretistisch versteht oder ob er sie als Beziehungs- und Veränderungswünsche begreifen kann und sich damit neue Handlungs- und Erlebensoptionen eröffnen. Erst in der sicheren und tragfähigen Beziehung zum Arzt realisieren sich die medizinethischen Werte Autonomie, Nichtschädigung und Fürsorge. Das erfordert die Bereitschaft, sich über eine längere Zeit in die Beziehung hineinziehen zu lassen und in ihr die Auseinandersetzung mit dem Patienten zu suchen. Nochmals Dornberg: «Erst nach dieser Zeit geteilten Lebens kann eine ethisch verantwortbare Entscheidung zur Frage der Sterbehilfe getroffen werden.»

Diese Art der beziehungsorientierten Hausarztmedizin deckt sich weitgehend mit den Zielen der Hospizbewegung und der Palliativmedizin, die sich, von anderen Seiten kommend, ebenfalls mit einem möglichst würdevollen und leidarmen Sterben befassen. Sie belässt den Sterbenden so weit möglich in seinem häuslichen Umfeld und erspart ihm damit unnötiges Leid. Cicely Saunders hatte auf die Defizite der damaligen Biomedizin reagiert und 1967 in London das St Christopher's Hospice gegründet. Die dadurch angestossene Hospizbewegung, die sich der medizinisch und pflegerisch kompetenten Begleitung von Sterbenden widmet, hat nach einer ersten Phase, in der eine stationäre Versorgung in Hospizen im Vordergrund stand, das Sterben zuhause gefördert $[1,12]$. Damit wird das Sterben zurück in den Alltag und in die vertrauten Strukturen der Sterbenden gebracht. Die Öffentlichkeit von Sterben, Trauer und Tod waren die wichtigen Elemente der Rituale früherer Jahrhunderte, die bei der Bewältigung der Angst vor dem Tod und des Verlustes halfen. Es braucht deshalb wieder eine Kultur des Sterbens - eine Ars Moriendi [13].

Die Möglichkeit der Fehlentwicklungen ist nicht in der Sterbebegleitung selbst begründet, sondern darin, dass der Mensch nicht mehr im Mittelpunkt steht, also verzwecklicht werden kann. Auch eine generelle Ablehnung von Sterbehilfe befreit nicht aus den ärztlichen Zielkonflikten des Lebensendes, unzumutbare Härten im Einzelfall bei möglichst umfassendem Schutz des Lebens und der Berücksichtigung eigener Grenzen zu vermeiden. Oberstes Gebot sollte deswegen die Ausrichtung auf den Menschen und auf seine Würde sein. Zur möglichst optimalen Behandlung sollte der einzelne Arzt die Möglichkeit besitzen, im Sinne einer ganzheitlichen Beziehungsmedizin seinen Patienten auch am Lebensende zu begleiten und ihm dabei die ganze Bandbreite der modernen Palliativmedizin anzubieten, aber ihm in seltenen Ausnahmefällen bei einem sorgfältig erwogenen Bilanzsuizid zur Seite zu stehen. Dies würde die Lebens- und Behandlungsqualität Sterbender verbessern und das Vertrauen der Patienten in die Ärzte stärken. Sie stellt jedoch eine grosse Herausforderung für das ärztliche Ethos jedes Einzelnen dar und kann nicht als postuliertes Recht eingefordert oder gar erzwungen werden [14]. Auf ärztlicher Seite erfordert es eine Auseinandersetzung mit der eigenen Angst vor dem Tod mit dem Ziel einer zumindest partiellen Distanzierung. Ausserdem muss der ärztliche Blickwinkel erweitert werden: Auch der Sterbende darf nicht auf seine biomedizinische Dimension reduziert werden. Seine kommunikativen und geistigen Dimensionen müssen mitberücksichtigt werden [15]. Um ihrer Verantwortung gegenüber der Gesellschaft gerecht zu werden, sollte sich die Ärzteschaft deshalb in interdisziplinären Gremien daran beteiligen, die Grundlagen für die Beihilfe zum Suizid innerhalb wie ausserhalb des Medizinsystems zu erarbeiten. Dadurch können modellhaft Standards entwickelt werden, an denen sich der Hausarzt in der relativ abgeschirmten Arzt-Patienten-Beziehung orientieren kann. Beide Wege können sich so ein Stück weit Korrektiv gegenüber systemimmanenten Tabuisierungen, Fehlentscheidungen und Fehlanreizen sein. 